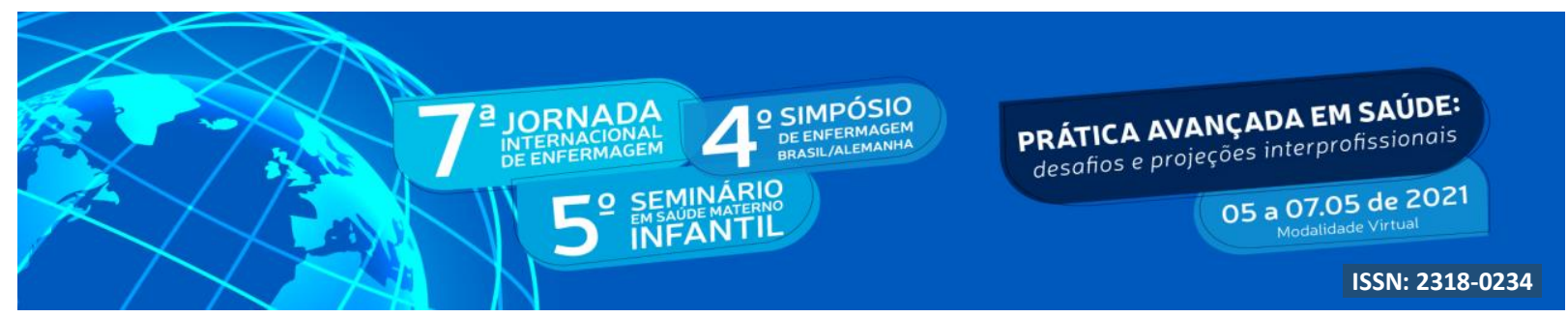

DOI: http://doi.org/10.48195/jie2021-142

\title{
STATUS SOCIOECONÔMICO E IMUNOMETABOLISMO: UMA PERSPECTIVAS INTERSECCIONISTA PARA A COMPREESÃO DA PREVALÊNCIA DA OBESIDADE ${ }^{1}$
}

\section{Karina Medeiros dos $\operatorname{Santos}^{1}$ Francisco Daroda Dutra ${ }^{2}$ Cristina Machado Bragança de Moraes $^{3}$}

RESUMO

A obesidade como uma epidemia global tras consequências econômicas, sociais, institucionais e individuais aos acometidos. A doença apresenta um padrão epidemiológico de aumento em países em desenvolvimento, No entanto, o modelo intervencionista pautado no gasto e ingestão para homeostase energética tem falhado a longo prazo, prevalecendo individualmente o peso adquirido e repassado intergeracionalmente. Um forte preditor de prevalência da obesidade é o Status Socioecônomico (SES), o qual é acompanhado pela piora de marcadores imunometabólicos, os quais pioram o tempo de vida humano e predizem a neurodegeneração. Para isso, revisou-se na literatura se há intersecção entre o imunometabolismo e o SES buscando elucidar futuras vias para uma terapeutica individualiza com maior sucesso na intervenção e redução da prevalência ambiental da obesidade.

Palavras-chave: Obesidade; Metabolismo; Status Socioeconômico (SES); Imunometabolismo; Inflamação; Curso da vida.

\begin{abstract}
Obesity as a global epidemic has economic, social, institutional and individual consequences for those affected. The disease has an epidemiological pattern of increase in developing countries. However, the interventionist model based on expenditure and intake for energy homeostasis has failed in the long term, prevailing individually and passed on intergenerationally. A strong predictor of obesity prevalence is Socioeconomic Status (SES), which is accompanied by worsening immunometabolic markers, which worsen human lifespan and predict neurodegeneration. For this, it was reviewed in the literature if there is an intersection between immunometabolism and SES seeking to elucidate future ways for an individualized therapy with greater success in the intervention and reduction of the environmental prevalence of obesity.
\end{abstract}

Keywords: Obesity; Metabolism; Socioeconomic Status (SES); Immunometabolism; Inflammation; Lifespan.

\footnotetext{
${ }^{1}$ Projeto dos alunos do Curso de Nutrição da Universidade Franciscana (UFN).

${ }^{2}$ Acadêmicos do Curso de Nutrição da Universidade Franciscana (UFN). Email: k.medeiros@ ufn.edu.br francisco.datoda@ufn.edu.br

${ }^{3}$ Professor orientador do trabalho e docente do curso de Nutrição e do Mestrado em Ciências da Saúde e da Vida da Universidade Franciscana (UFN). Email: cbmoraes@ $\mu$ fn.edu.br
} 


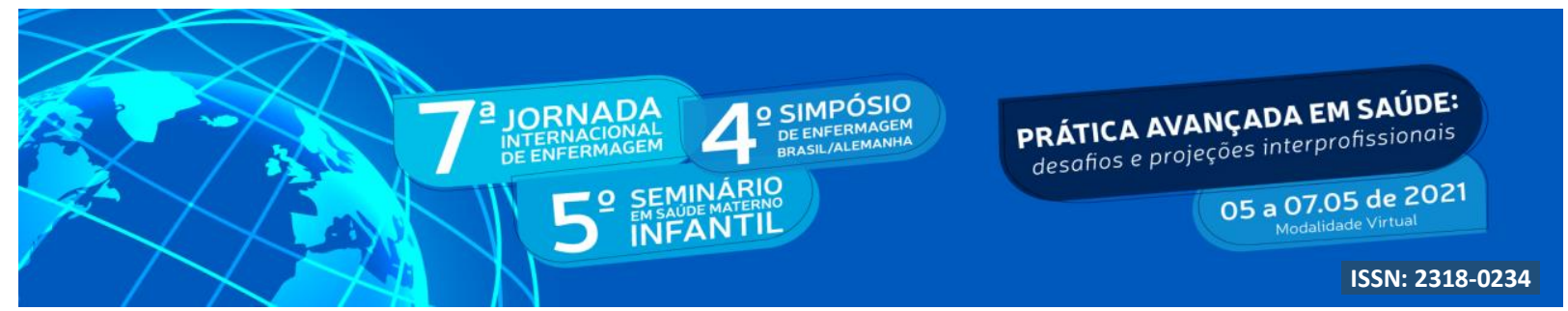

\section{INTRODUÇÃ̃}

A Obesidade é um problema mundial, o qual segue-se em crescimento no mundo em incidência e prevalência, com o aumento acentuado nas américas, inclusive no Brasil (CHOOI et al., 2019). A obesidade é categorizada, pela Organização mundial da Saúde, através de um Índice de Massa Corporal (IMC) maior do que $30>\mathrm{Kg} / \mathrm{m}^{2}$ o qual é mensurado a partir do peso do indivíduo dividido pela altura em metros quadrado (OMS, 2020).

Essa condição é evitável devido a sua etiologia encontrar-se no crônico consumo de energia acima do gasto energético, no entanto, causalmente é desencadeada de forma multifatorial e precedido por mudanças econômicas-culturais e hereditárias (SWINBURN et al., 2011; MATTHIAS, 2019).

Um dos fatores que moldam o contexto ambiental é o Status Socioeconômico (SES) que pode ser considerado uma medida objetiva e subjetiva, também chamada de Status Socioeconômico Subjetivo (SSS). Objetivamente o SES está relacionado a piores cuidados com saúde e aquisição de alimentos de baixa qualidade nutricional (ALLEN et al., 2017).

Não obstante, adversidades no início da vida, como perceber-se socialmente inferior, podem predispor a uma resposta subsequente inflamatória amplificada, em ratos e humanos (SAXTON et al., 2011). Além do ambiente fetal e o status socioeconômico da mãe durante a gestação associarem-se a sobrepeso e obesidade adjacente (ZHANG et al., 2021) e mais severa resposta inflamatória a eventos agudamente estressantes (LOOCKWOOD et al., 2018). O SES também prediz o aumento de adiposidade central em minorias étnicas e níveis de interleucina-6 (IL-6) elevados (LAM et a.1, 2021).

Logo, o presente trabalho visa clarificar a possível associação entre o Status Socioeconômico como um preditor para a resposta imunológica inflamatória, e construir uma ligação entre a teoria biológica da regulação energética para ingestão e gasto, que atualmente pauta a dificuldade na perda e manutenção de peso em indivíduos obesos, socialmente desprivilegiados. 


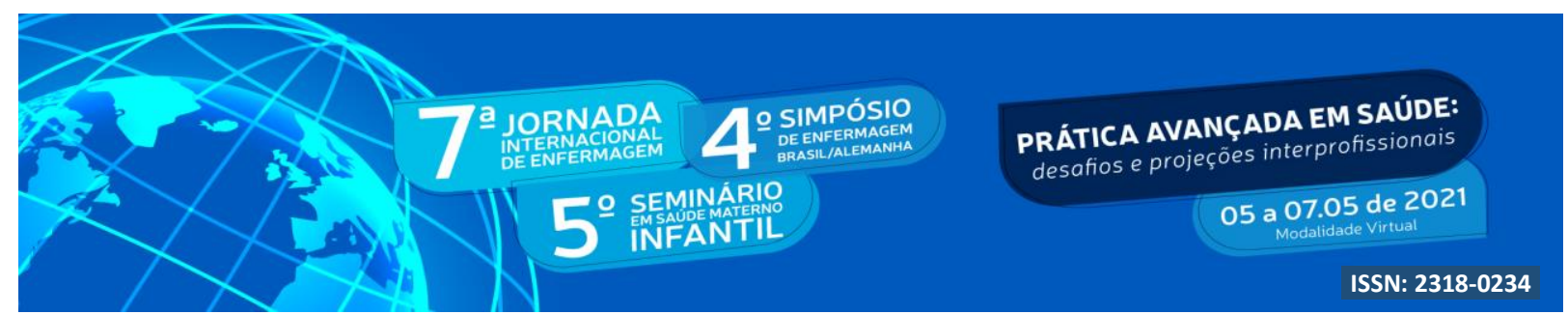

\section{OBJETIVO}

Elaborar uma revisão literária, baseada no conhecimento técnico científico referente as variáveis que interferem e/ou dificultam a redução do peso corporal em indivíduos acima do peso, tais quais, classificados como sobrepeso e obesidade.

\section{METODOLOGIA}

Realizar-se-á uma pesquisa na literatura utilizando as palavras-chaves que norteiam o corpo do texto: Obesidade; Metabolismo; Status Socioeconômico (SES); Imunometabolismo; Inflamação; Curso da vida, e tal pesquisa poderá envolver mais de uma palavra chave como marcador de busca unitário.

\section{RESULTADOS E DISCUSSÃO}

\section{Status Socioeconômico - Variáveis objetivas e subjetivas na obesidade}

Um dos fatores que moldam o contexto ambiental é o Status Socioeconômico (SES), o qual pode ser definido por variáveis objetivas como: renda, escolaridade, vizinhança e ocupação. Subjetivamente a percepção da posição social também serve como uma variável para que indivíduos se percebam inferiores aos demais, a qual cunhada sobre Status Socioeconômico Subjetivo (SSS) (NOBLES et al., 2013).

Tal medida correlaciona-se a piores prognósticos em saúde (OPERARIO et al., 2004) e maior circunferência de cintura, HDL-colesterol e maior risco de Diabetes Mellitus tipo 2 (DM2) em mulheres (DEMAKAKOS et al., 2008). Subjetivamente, perceber-se inferior socialmente relaciona-se a piores marcadores metabólicos e consumo de alimentos mais densos em energia e em maior quantidade (CHEON; HONG, 2017).

\section{Curso da vida, obesidade e metabolismo no contexto socioeconômico}

A carga de doenças é um marcador comum entre indivíduos socioeconomicamente desfavorecidos, os quais apresentam maior prevalência de múltiplas comorbidades 


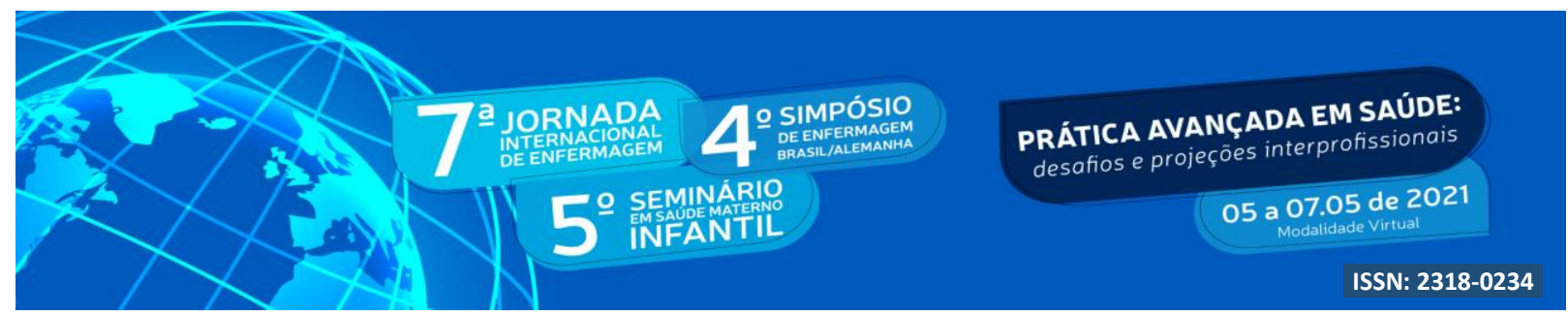

(SINGH MANOUX et al., 2018). Além disso, o fardo socioeconômico estende-se para associação com múltiplas morbidades como o consumo de álcool, tabagismo, inatividade física (KIVIMAKI et al., 2020). E a presença de multimorbidades associa-se à maior vulnerabilidade e menor adesão a hábitos alimentares e de atividade física, principalmente em mulheres idosas de uma coorte coreana (PARK et al., 2018).

Sequencialmente, a obesidade e as múltiplas comorbidades convergem para o encurtamento de vida, maior resistência à insulina, inadequada autofagia, apoptose celular e ao encurtamento dos telômeros (TAM et al., 2020). O que torna necessário a visão integrativa da piora metabólica e a descompensação de um indivíduo em direção da não reversão do estado de saúde (RASGON; MCEWEN, 2016). Não obstante, modelos preditivos regulação alostática foram propostos para a antecipação de um organismo as adversidades ambientais, inclusivamente na resposta imune a mecanismos circadianos moleculares (SHULKIN; STARLING, 2019).

Portanto, parece prudente interseccionar áreas do conhecimento no desenvolvimento de uma terapêutica pautada na individualização, levando em consideração fatores além da perspectiva de entrada e saída de energia no tratamento da obesidade.

\section{Imunometabolismo, pressão socioambiental e regulação energética}

Foi demonstrado que a obesidade leva a danos funcionais no tecido adiposo (TA). O TA sofre constante adaptação para atender a demanda ambiental e interage com a imunidade migratória e residente (CREWE, et al., 2017) através de citocinas, quimiocinas e hormônios.

O tecido adiposo de um obeso tem alteração metabólica e endócrina, com uma maior secreção de citocinas pró-inflamatórias (WEISBERG, et al., 2003) as quais moldam a hipertrofia e hiperplasia tecidual podendo estender a condição conhecida como inflamação sistêmica de baixo grau (FJELDBORG, et al., 2014).

Além de perdurar no TA através de promotores epigenéticos, TNF- $\alpha$ e IL-6, exacerbando a apoptose adiposa moldando fenotipicamente macrófagos e monócitos migratórios a uma população simplisticamente conceituada como M1 ou pró-inflamatórios (LUFT et al., 2013; DALMAS et al., 2011). A qual diferencia-se do outro extremo fenotípico M2, caracterizado pela secreção de IL-4 e IL-10 (DALMAS et al., 2011). 


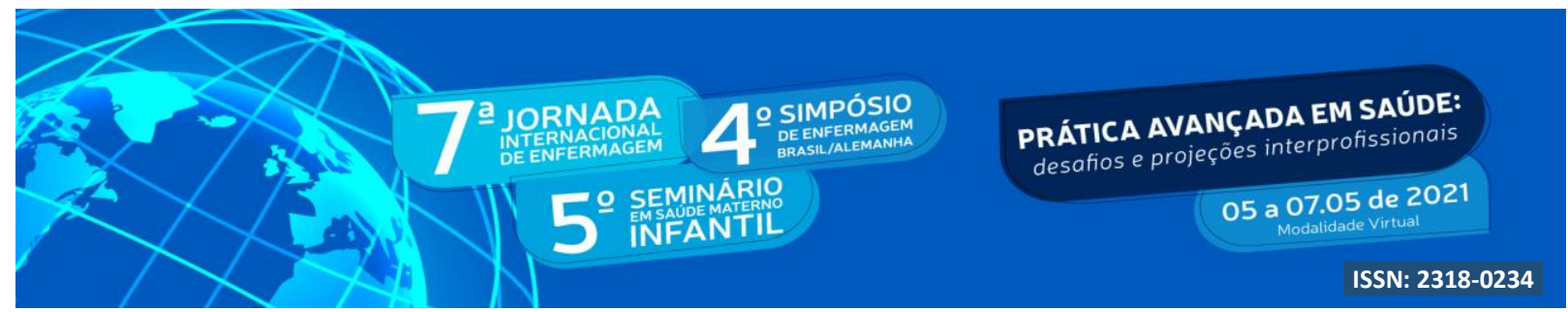

Ademais, há mudanças morfológicas no TA com aumento da quimiotaxia e adesão macrofagica levando a formação de uma "estrutura em formato de coroa", a qual exacerba a hipóxia tecidual (ZHENG et al., 2016). As mudanças teciduais adiposas, consequentes da obesidade também estão associadas a outras patologias como: Doença Hepática Gordurosa não Alcoólica, Hipertensão Arterial Sistêmica, Osteoartrite, Acidente Vascular Cerebral, Apneia Obstrutiva do Sono, DM2 e diversos tipos de câncer, reduzindo a qualidade e a expectativa de vida (MATTHIAS, 2019).

Não obstante, a obesidade e o DM2 são associados ao menor SES e a inflamação crônica de baixo grau (STRIGHINI et al., 2013), com crescente impacto em populações desfavorecidas em SES, coadunado a hábitos alimentares inadequados, desconhecimento da própria patologia e difícil acesso a serviços de saúde (PSALTOPOULOU et al., 2017). Sobretudo, a RI pode encurtar o tempo de vida do indivíduo e ser o elo entre os distúrbios afetivos, obesidade e demência (RASGAN; MCEWEN, 2016).

Conjuntamente, apenas atualmente cresce as pesquisas relacionando epigenética, ambiente e metabolismo, com o status socioeconômico subjetivamente e objetivamente relacionado a pior controle do peso (BLÜHER et al., 2016). Além da inadequada valoração a pistas alimentares, menor autoregulação frente a recompensa alimentar, e menor perspectivas em saúde encontrada em indivíduos obesos com menor SES (LOWE et al., 2019). Não obstante, os modelos preditivos de homeostase energética não levam em consideração a relação do indivíduo com o ambiente e suas antecipações ingestivas e no gasto calórico (CHAPELOT; CHARLOT, 2018).

Finalmente, indivíduos com baixo SES e em áreas pobres apresentam maiores determinantes para obesidade e níveis maiores de marcadores inflamatórios (MUSCATELL et al., 2018; PSALTOPOULOU et al., 2017). O que tende a se perpetuar entre gerações em um mesmo ambiente, prevalecendo em elevados indices de obesidade (ZHENG et al., 2016). E parece depender de intervenções promotoras de exercício físico, adequação alimentar e redução de outros comportamentos potencialmente nocivos como: alcoolismo, tabagismo (ROHDE et al., 2018). 


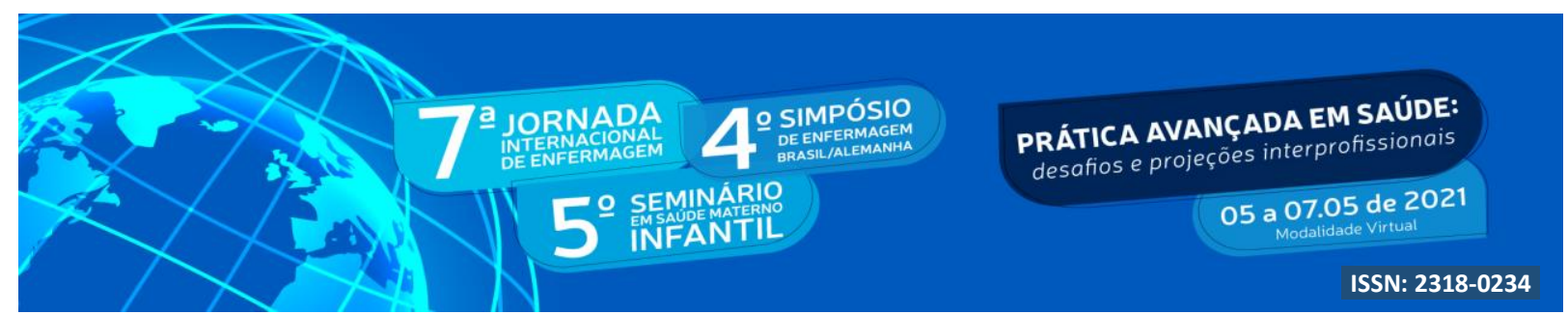

\section{Conclusão e Perspectivas}

O status socioeconômico parece acompanhar a prevalência da obesidade conjuntamente ao aumento de biomarcadores inflamatórios em indivíduos obesos, ambos são independentemente associados a um pior prognóstico para indivíduos obesos e merecem ser incluídos na prática clínica de forma a individualizar o tratamento da obesidade.

\section{REFERÊNCIAS}

CHAPELOT, D. et al. Fisiologia da homeostase energética: modelos, atores, desafios e a alça glicoadipostática. Metabolism, 2018).

CHEON, B. K. et al. A mera experiência de baixo status socioeconômico subjetivo estimula o apetite e a ingestão de alimentos. Proceedings of the National Academy of Sciences, 2016.

CHOOI, Y. C. et al. A epidemiologia da obesidade. Metabolism: Clinical and Experimental, 2019.

DALMAS, E. et al. Definição do fenótipo e função dos macrófagos no tecido adiposo. Trends in Immunology, 2011.

FRANÇA, E. B. et al. Mortalidade por causas específicas para 249 causas no Brasil e estados durante 1990-2015: uma análise sistemática para o estudo da carga global de doenças de 2015. Population Health Metrics, 2017.

HULSHOF, K. et al. Status socioeconômico, ingestão alimentar e tendências de 10 anos: Pesquisa Nacional de Consumo de Alimentos da Holanda. European Journal of Clinical Nutrition, 2003.

KIVIMÄKI, M. FM. et al. Associação entre nível socioeconômico e desenvolvimento de condições de saúde mental e física na vida adulta: um estudo multicorte. The Lancet Public Health, 2020.

LAM, P. H. et al. Raça, status socioeconômico e biomarcadores inflamatórios de baixo grau ao longo da vida: uma análise conjunta de sete estudos. Psychoneuroendocrinology, 2020.

LOWE, C.J. et al. O Córtex Pré-Frontal e a Obesidade: Uma Perspectiva da Neurociência em Saúde Tendências em Ciências Cognitivas, 2019. 


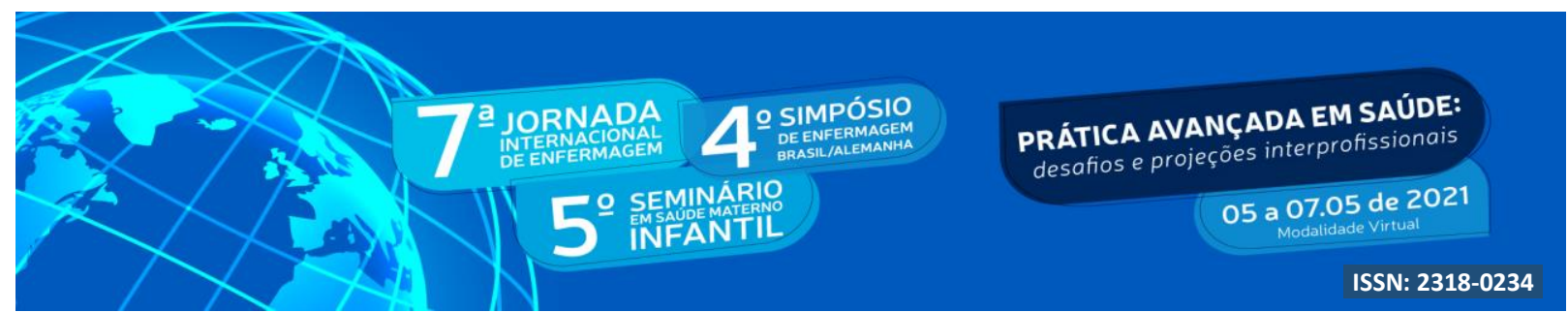

LUFT, V. C. et al. Papel da inflamação crônica na associação obesidade-diabetes: um estudo de caso-coorte. Diabetol Metab Syndr, 2013.

MARTIKAINEM, P. et al. Diferenças socioeconômicas nos padrões alimentares entre homens e mulheres de meia-idade. Social Science \& Medicine, 2003.

MUSCATELL, K. A, et al. Status socioeconômico e inflamação: uma meta-análise. Molecular Psychiatry, 2018.

NOBLES, J. et al. Status socioeconômico subjetivo e saúde: relacionamentos reconsiderados. Social Science \& Medicine, 2013.

OPERARIO, D. et al. Status social subjetivo: confiabilidade e utilidade preditiva para a saúde global. Psychology \& Health, 2004.

Organização Mundial da Saúde. Obesidade e excesso de peso, 2020.

PARK, B. et al. Multimorbidade e qualidade de vida relacionada à saúde em coreanos com 50 anos ou mais usando o KNHANES 2013-2014. Resultados de Saúde Qual Life, 2018 .

PSALTOPOULOU, T. et al. Status socioeconômico e fatores de risco para doenças cardiovasculares: Impacto dos mediadores dietéticos. Hellenic Journal of Cardiology, 2017. RASGON, N. L. et al. Resistência à insulina - um elo perdido não mais. Mol Psychiatry, 2016.

ROHDE, K. et al. Genética e epigenética na obesidade. Metabolism, 2018.

SAXTON, K. B. et al. O ambiente social e a IL-6 em ratos e humanos. Brain, Behavior, and Immunity, 2011.

SCHULKIN, J. et al. Alostase: Um modo de regulação fisiológica centrado no cérebro e preditivo. Trends in Neurosciences, 2019.

SINGH-MANOUX, A. et al. Fatores clínicos, socioeconômicos e comportamentais na idade de 50 anos e risco de multimorbidade cardiometabólica e mortalidade: Um estudo de coorte. PLOS Medicine, 2018.

STRINGHINI, S. et al. Associação do status socioeconômico ao longo da vida com inflamação crônica e risco de diabetes tipo 2: Estudo de coorte prospectivo de Whitehall II. Prospective Cohort Study. PLoS Medicine, 2013.

SWINBURN, B. MD. Et al. A pandemia global de obesidade: moldada por motivadores globais e ambientes locais. The Lancet, 2011. 


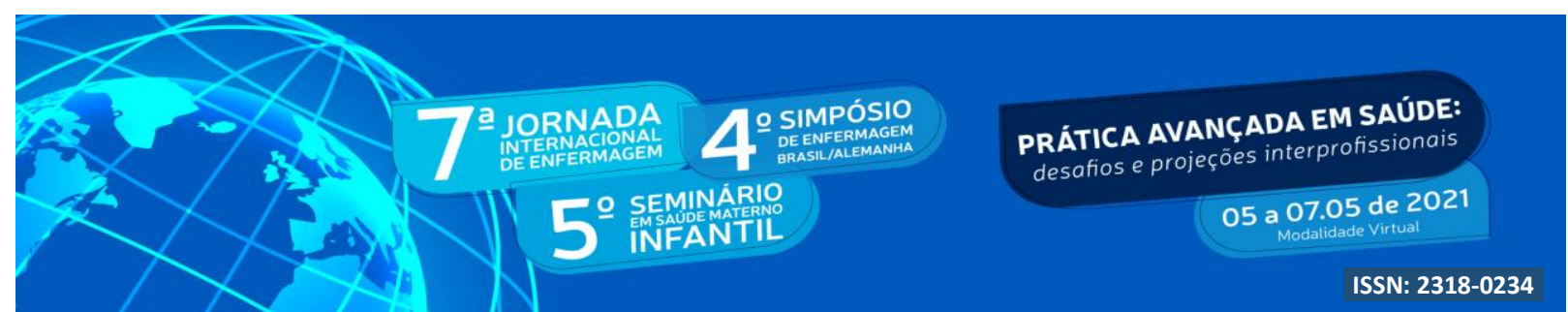

TAM, B. T. et al. Obesidade e envelhecimento: duas faces da mesma moeda. Obesity Reviews, 2020.

WEUHRAUCHU, S. B. et al. Regulação do peso corporal, nível socioeconômico e alterações epigenéticas. Metabolism, 2018.

WEXLER, D. J. et al. Efeitos mediadores de biomarcadores inflamatórios na resistência à insulina associada à obesidade. Obesity Research, 2005.

YUANYUAN, Z. et al. Associação e potenciais mediadores entre status socioeconômico e sobrepeso/obesidade infantil, Preventive Medicine, 2021.

ZHENG, C. et al. A proliferação local inicia o acúmulo de macrófagos no tecido adiposo durante a obesidade. Cell Death Disease, 2016. 